This item was submitted to Loughborough's Research Repository by the author.

Items in Figshare are protected by copyright, with all rights reserved, unless otherwise indicated.

\title{
Tunable resistive pulse sensing: potential applications in nanomedicine
}

\section{PLEASE CITE THE PUBLISHED VERSION}

http://dx.doi.org/10.2217/nnm-2016-0097

\section{PUBLISHER}

(c) Future Medicine

\section{VERSION}

AM (Accepted Manuscript)

\section{PUBLISHER STATEMENT}

This work is made available according to the conditions of the Creative Commons Attribution-NonCommercialNoDerivatives 4.0 International (CC BY-NC-ND 4.0) licence. Full details of this licence are available at: https://creativecommons.org/licenses/by-nc-nd/4.0/

\section{LICENCE}

CC BY-NC-ND 4.0

\section{REPOSITORY RECORD}

Sivakumaran, Muttuswamy, and Mark Platt. 2019. "Tunable Resistive Pulse Sensing: Potential Applications in Nanomedicine". figshare. https://hdl.handle.net/2134/22384. 


\section{Tunable Resistive Pulse Sensing: Potential Applications in Nanomedicine}

Muttuswamy Sivakumaran ${ }^{1 *}$ Mark Platt $^{2}$

1. Department of Haematology, Peterborough City Hospital, Peterborough, PE3 9GZ, UK

2. Department of Chemistry, Loughborough University, Loughborough, LE11 3TU, UK.

\section{* contact info and your email as corresponding author?}

\section{Abstract}

An accurate characterisation of nanomaterials used in clinical diagnosis and therapeutics is of paramount importance to realise the full potential of nanotechnology in medicine and to avoid unexpected and potentially harmful toxic effects due to these materials. A number of technical modalities are currently in use to study the physical, chemical and biological properties of nanomaterials but they all have advantages and disadvantages. In this review, we discuss the potential of a relative newcomer, Tunable Resistive Pulse Sensing (TRPS), for the characterisation of nanomaterials and its applications in nanodiagnostics.

Keywords: Tunable Resistive Pulse Sensing, TRPS, nanotechnology, nanomedicine, nanodiagnostics, nanoparticles, aptamers, exosomes, biosensors.

\section{Introduction}

Nanomedicine, a branch of Nanotechnology, despite it being a relatively new discipline, has already shown great promise in the areas of in-vitro medical diagnostics, in-vivo imaging, regenerative medicine, drug development and delivery [1-3]. The unique properties of nanomaterials (size, high surface to volume ratio, surface characteristics and functionality) make them ideally suited for biosensors and for targeted drug delivery. The size of the nanomaterial gives a distinct advantage in that a drug could be transported across physiological barriers such as blood brain barrier. Furthermore, the relative ease by which nanomaterials could be linked with various biomolecules such as antibodies or ligands, to precisely target and deliver a drug to a particular organ or tissue is a major advantage of this technology. Such precise targeting can greatly reduce systemic toxicity. Another unique feature of this technology is that a nanopharmaceutical could be constructed to carry not only a drug but also a sensor to enable visualisation of the diseased tissue by imaging and also achieving targeted delivery of a drug. This combined therapeutic and diagnostic application (dubbed 'Theranostics') is fast becoming a reality.

It must, however, be emphasised that despite the great potential of nanomaterials in medicine, in order to realise their full potential, they must be fully characterised [4]. The size, shape and surface charge of the nanomaterials must be accurately analysed. In addition, detailed evaluation to study the fate of nanopharmaceuticals following systemic administration must be carried out. These evaluations must include stability of the nanomaterial in body fluids and tissues i.e. whether they undergo any disintegration in biological fluids that may result in significant change to their properties. Conversely the nanomaterial may coalesce to form larger particles with different properties or form aggregates with altered functions. In addition, 
detailed analysis into how nanopharmaceuticals interact with the components of body fluids such as blood must be carried out. In particular, the interaction with various plasma proteins, lipids and other biomolecules, change in the surface charge (zeta potential) and the 'corona' effect must be studied. Methods to study interaction of nanopharmaceuticals with various blood cells to find out whether they passively adsorb onto cells or actively bind to a cell surface due to upregulation of various cellular adhesion molecules, whether the nanomaterial causes significant activation of any cell (eg. neutrophils, monocytes, lymphocytes, platelets and vascular endothelial cells) and whether the nanomaterial causes any cell membrane damage resulting in increased permeability or cell lysis must be established.

There is a range of techniques that are being used to study the physico-chemical characteristics of various nanomaterials (Box 1). They all have advantages and disadvantages and not a single technique that will be able provide all the information required exists. A recent addition to this diagnostic array is Tunable Resistive Pulse Sensing which is a variant of the much established technology of Resistive Pulse Sensing (RPS). 


\section{Analytic techniques for characterisation of nanomaterials}

\section{Microscopy}

High resolution Optical Microscope

Confocal Microscope

Multiphoton Microscope

4Pi Microscope

Cyto Viva Microscope

Magnetic Resonance Force Microscope (MRFM)

Fluorescence Microscope

Scanning Electron Microscope (SEM)

Transmission Electron Microscope (TEM)

Freeze Fracture Transmission Electron Microscope (ffTEM)

Scanning Tunnelling Microscope (STM)

Atomic Force Microscope (AFM)

Scanning Near-Field Optical Microscope (SNOM)

\section{Resistive Pulse Sensing}

High-resolution flow cytometry

\section{Diffraction techniques}

X-ray Defraction

Electron Defraction

Neutron Defraction

Small Angle $X$ ray Scattering (SAXS)

Small Angle Neutron Scattering (SANS)

Dynamic Light Scattering (DLS)

\section{Nanoparticle Tracking Analysis (NTA)}

\section{Spectroscopy}

Optical absorption spectrometer

UV spectrometer

Infrared spectrometer

Dispersive infrared spectrometer

Fourier transform infrared spectrometer

Raman spectroscopy

UV photoelectron spectroscopy

Auger electron spectroscopy

Magnetic measurements

Vibrating sample magnetometer (VSM)

Box 1. Analytic techniques for characterisation of nanoparticles.

(Source: Kulkarni SK [5], Jain KK [2]) 


\section{Resistive Pulse Sensing}

The technique of resistive pulse sensing (RPS) to enumerate particles in solution was first developed by Wallace. H. Coulter in early 1950s [6]. The principle of this technique is that when a particle traverses through a channel filled with aqueous electrolyte solution, there is a transient change in the channel's ionic resistance known as a 'resistive pulse'. As envisioned by Coulter, this technology was soon adopted for rapid counting of particles in biological fluids in particular in the field of Haematology where it is extensively used for enumeration of blood cells. Subsequently, in the 1970s, this technique was adapted for counting microorganisms including viruses and bacteriophages by de Blois and colleagues [7]. They were able to accurately size several type $\mathrm{C}$ oncorna viruses and T2 bacteriophages with a detection limit of $5 \times 10^{7}$ viruses per $\mathrm{ml}$.

A landmark development of a method to detect single stranded DNA using biological alpha-haemolysin pore by Kasianowicz et al in 1996 [8] with a promising potential of DNA sequencing reignited interest in resistive pulse sensing technology. This development together with the on-going rapid growth in nanotechnology based research and applications resulted in the resurgence of RPS based techniques for particle characterisation [9-11].

A variety of materials including carbon nanotubes [9], polydimethylsiloxane (PDMS) (Saleh OA \& Sohn LL, 2002), glass (Lan et al, 2011], silicon [12] and polycarbonate (Sexton LT et al, 2007; Lee et al, 2004) have been used in RPS devices.

One of the major attributes of the RPS technology is its ability to provide valuable information on individual particles within their environment [12-13]. Hence, this technology has been used to study a variety of parameters including concentration, size, charge and conductivity of diverse type of particles, naturally occurring such as peptides, proteins, nucleic acids, cell derived particles (exosomes) and biomolecular complexes or man-made organic or inorganic nanomaterials, in different situations with exquisite resolution [9, 11-12, 14-16].

\section{Tunable Resistive Pulse Sensing (TRPS)}

TRPS is an adaptation of RPS in which utilises a conical tunable elastomeric pore that can be stretched or relaxed to change the pore size to suit the sample $[11,13$, 15-19]. Tunable pore membranes are made of thermoplastic polyurethane and the pores are introduced by mechanical puncture. The concept of tunable nanopore membrane was first developed by Sowerby [20]. The membrane can be stretched in a biaxial direction to alter the pore size. The size of the pore can be altered as much as an order of magnitude [17].

\section{Principle of Tunable Resistive Pulse Sensing}

The instrument associated with TRPS is produced by the company Izon Science, and as with standard RPS equipment two fluid reservoirs are to be filled with a conducting electrolyte solution. In the setup the pore is mounted horizontally and 
reservoirs oriented above and below the pore membrane, with the sample typically placed into the top reservoir, see figure 1.
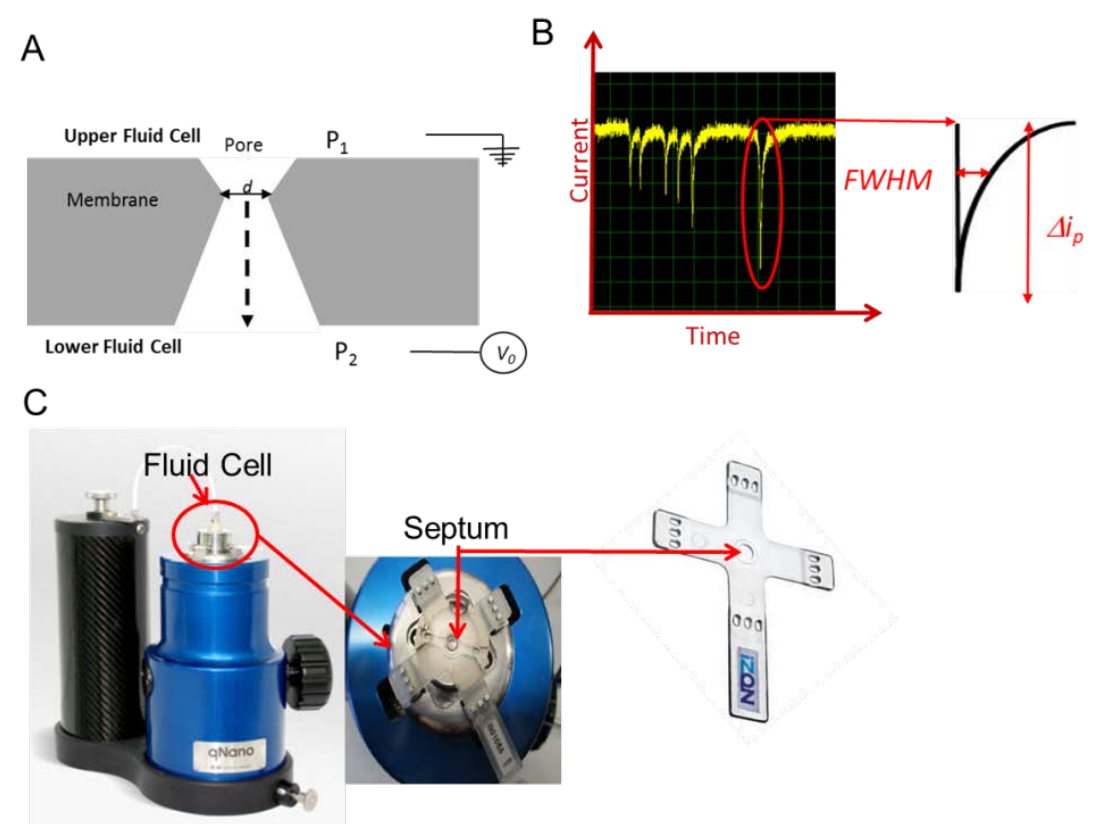

Figure $1 \mathrm{~A}$. Sectional schematic of a pore. The sample is typically placed into the upper fluid cell. B. Example of baseline current and "blockade" events (current dips) that are each caused by an analyte traversing the pore. Each event is analysed for full width half maximum (FWHM) duration and $\Delta i_{\mathrm{p}}$. C. The Izon qNano instrument, showing the fluid cell and location of crucifix plastic membrane.

\section{Applications of Tunable Resistive Pulse Sensing}

TRPS technology has a number of merits. The pore tunability offers flexibility in analysing particles of broad size range (40nm-10um). It enables particle by particle measurement of size and surface charge of individual particles simultaneously. it can also analyse particle aggregation and stability. It works with physiological strength buffers, provides high resolution data points, large number of data points and requires no software-induced data manipulation. It is relatively inexpensive off the shelf instrument requiring a few consumables and not needing carrier gases, fluidics or optics.

\section{TRPS in Nanoparticle Counting}

As particles/analytes translocate the pore they temporarily occlude ions, leading to a transient decrease in current known as a "blockade event", examples of which can be seen in figure $1 \mathrm{~b}$. The pulse frequency, $J$, is related to the concentration of the analyte, $C_{s}$, multiplied by the velocity of the traversing particle, $v_{p}$ (ignoring contributions from diffusion). The velocity term is the sum of the fluidic, $V_{F}$, electrophoretic, $v_{E}$, and electroosmotic, $v_{O}$, velocities, i.e. $v_{p}=v_{F}+v_{E}+v_{O}$. Here we typically ignore the contribution from diffusion due to the magnitude of other forces and end effects are not taken into account in the analysis. $v_{p}$ can be written as; 
$v_{p}=\frac{Q}{\pi\left(\frac{D_{S}}{2}\right)^{2}}+\frac{E \zeta_{\text {particle }}}{\eta} E-\frac{s \zeta_{\text {pore }}}{\eta} E$

Where $Q=\frac{3 \pi D_{s}^{\mathrm{g}} \Delta P}{128 \eta\left(\frac{L}{D_{L}-D_{S}}\right)}$

$\square$ and $\square$ are the permittivity of the solution and kinematic viscosity respectively, $\square P$ is the pressure across the pore, $\square_{\text {ore }}$ and $\square$ article are the zeta potential of the channel surface and particle respectively, and $E$ is the electric field.

\section{TRPS in Nanoparticle Size Analysis}

The pores in the TRPS system are conical in shape and give rise to an asymmetric current pulse, figure $1 \mathrm{~b}$ [21]. For a conical pore, the change in the resistance, $\Delta R$, across the length of the pore. $L$, is given by equation 2 [22].

$$
\Delta R=\rho \int_{0}^{L} \frac{d z}{A(z)}-R
$$

where $\rho$ is the resistivity of the electrolyte that is filling the pore, $A(z)$ is the cross sectional area perpendicular to the pore axis $z$ and $R$ is the pore resistance. When no blockage is present, $R$ is given by equation $2,<\sup >1</$ sup $>$

$$
R=\frac{4 \mathbb{L}_{\rho}}{\pi \mathbb{D}_{\mathbb{L}} \mathbb{D}_{\mathbb{S}}}
$$

Where $D_{L}$ and $D_{S}$ are the largest and small pore diameters. When a particle traverses through the pore, a blockade event is observed. This blockade is created by the particle displacing a volume of electrolyte which in turn increases the resistance in the circuit, temporarily lowering the current. The blockade magnitude can then be used to size the particles or analyte as the magnitude of the increased resistance is directly related to the size of the analyte.

It must be noted that, unlike solid state pores where the size of the pore is always known, the tunable pore must first be characterised before users can accurately determine the size of the analyte using TRPS. This is done easily using calibration beads of a known size with a narrow size distribution amongst a sample population, and must be done prior to sample analysis and under the same conditions.

A number of different techniques are available for the measurement of nanoparticle size (Box 1). Some of which (dynamic light scattering (DLS), small angle $X$ ray scattering (SAXS) and static light scattering (SLS)) are termed 'Batch Particle Size Measurement methods because they perform size dispersion analysis of particles on the whole population ('batch'). On the other hand, techniques such as microscopy including electron and atomic force microscopies, Nanoparticle Tracking Analysis (NTA) and TRPS carry out single particle by particle measurement and are collectively called Single Particle Size Measurement methods. In a recent study, Varenne and co-workers (ref) evaluated nine different techniques in carrying out particle size distribution (PSD) of multimodal dispersion of nanoparticles. Their study showed that while single particle size measurement methods accurately determined 
the PSD, none of the batch particle size measurement methods was able to characaterise the complex PSD (Figure 2)

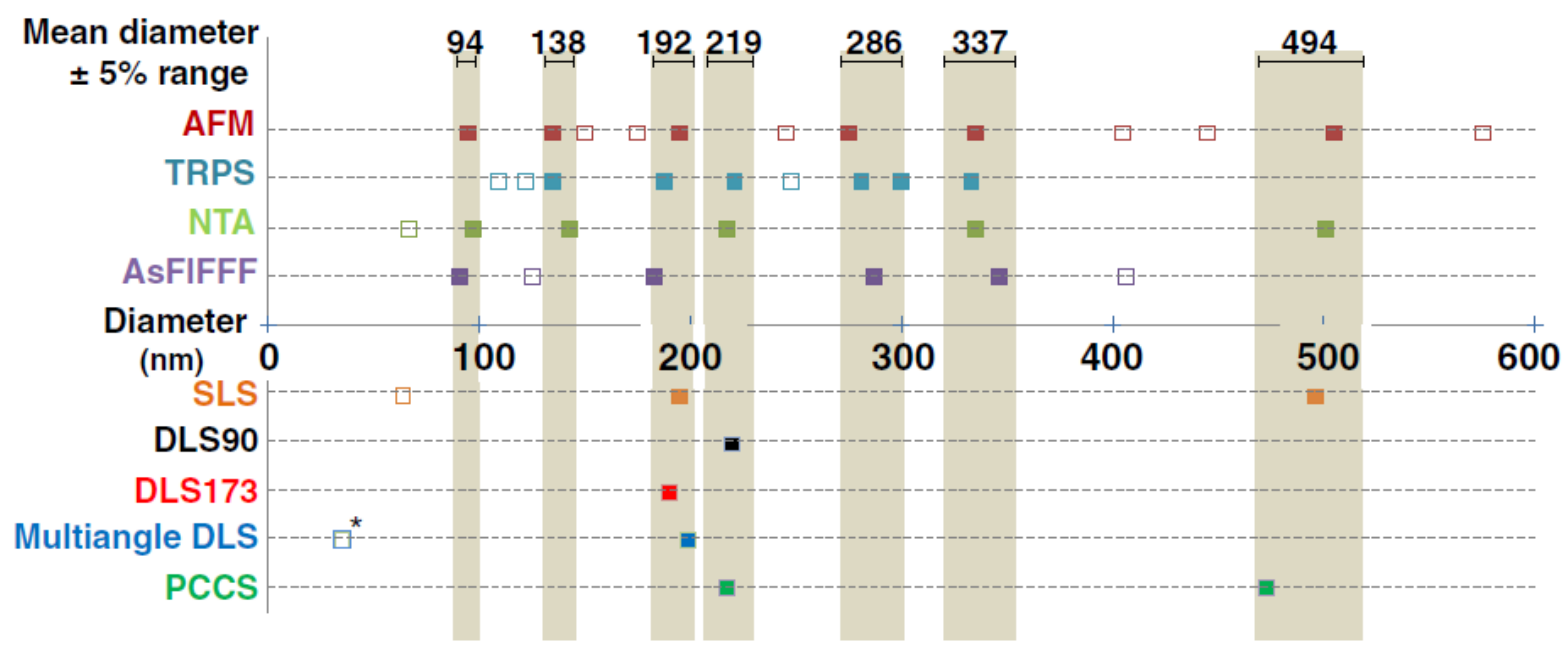

Figure 2. Summary of the obtained PSD from the dispersion of PIBCA nanoparticles analyzed by different size measurement methods. Results from measurements performed with single size measurement methods (AFM, TRPS, NTA) and methods based on a separative process (AsFIFFF) are shown on the upper part of the graph. Results from measurements performed with batch size measurement methods comprising different light scattering methods (SLS, DLS, PCCS) are summarized on the lower part of the graph. * indicates a significant variation of the size position of the corresponding detected population. Mean diameters were calculated from the diameters of the population of nanoparticles with the corresponding size that was detected in a $\pm 5 \%$ range (shown by the grey background) by at least 3 different methods. Reprinted with permission from Pherm. Res (2016), 33: 1220-1234. Copyright Springer Science Business Media New York 2016

\section{TRPS in analysis of shape and two/three dimensional geometric structures of nanoparticles}

The majority of the work published to date has focused on spherical analytes or particles. Some initial studies by Willmott and Platt [18] have shown that some information of length of asymmetric particles (cylinders) or clusters of spherical particles can be extracted from peak width/ pulse duration. Other groups have also used the flexible TRPS pore to deform and characterise soft polymer like particles [23].

\section{TRPS in analysis of particle aggregation}

Analysis of particle aggregation forms the basis of a number of diagnostic platforms including biosensors and point of care devices. TRPS has been used successfully in the detection of very low concentrations of analytes because of its ability to detect and enumerate particle aggregates that are formed as a result of binding between 
the functionalised nanoparticles and the analyte. Examples of studies in which TRPS has been used to detect nanoparticle aggregation are given in Table 1.

\begin{tabular}{|c|c|c|c|c|c|}
\hline $\begin{array}{l}\text { Particle } \\
\text { material }\end{array}$ & Particle size $(\mathrm{nm})$ & Surface function & Analyte & $\begin{array}{l}\text { Analyte } \\
\text { concentration }\end{array}$ & Reference \\
\hline Au/Ni rods & $\begin{array}{l}1230 \text { long } x \\
300 \text { diameter } \\
1100 \text { long } x \\
300 \text { diameter }\end{array}$ & $\begin{array}{l}\text { Avidin (Ni) and } \\
\text { PEG (Au) } \\
\text { Aptamer (Ni) and } \\
\text { PEG (Au) }\end{array}$ & $\begin{array}{l}\text { Biotin } \\
\text { PDGF }\end{array}$ & $\begin{array}{l}\sim 100 \mathrm{fM} \\
\sim 100 \mathrm{fM}\end{array}$ & 67 \\
\hline SPBS & $\begin{array}{l}3000 \\
1000 \\
300\end{array}$ & $\begin{array}{l}\text { Avidin } \\
\text { Avidin } \\
\text { Streptavidin }\end{array}$ & $\begin{array}{l}\text { Biotin } \\
\text { Biotin } \\
\text { Biotin }\end{array}$ & $\begin{array}{l}\sim 1 \mathrm{pM}-1 \mathrm{nM} \\
\sim 1 \mathrm{nM} \\
\sim 1 \mathrm{nM}\end{array}$ & 68 \\
\hline $\mathrm{Au}$ & 25 & DNA & DNA & $\sim 5 \mathrm{pM}$ & 69 \\
\hline $\mathrm{Au}$ & 50 & Citrate & PNA & $\sim 5 \mathrm{nM}$ & 73 \\
\hline $\mathrm{Au}$ & $\begin{array}{l}30 \\
35\end{array}$ & $\begin{array}{l}\text { Avidin } \\
\text { DNA }\end{array}$ & DNA & 530 copies & 98 \\
\hline $\begin{array}{l}\text { Magnetic } \\
\text { beads }\end{array}$ & 1000 & Protein & RBCs & $\sim 10^{7}$ per $\mathrm{ml}$ & 99 \\
\hline
\end{tabular}

Table 1. Particle aggregation studies using TRPS.

$P E G=$ polyethylene glycol, $P D G F=$ platelet derived growth factor, $P N A=$ peptide nucleic acid, SPBs = superparamagnetic beads, $R B C s=$ red blood cells. Adapted from Weatherall \& Willmott, Analyst, 140, 3318-3334 (2015). [ref]

\section{TRPS in nanoparticle surface charge analysis}

Evident from equation 3 is the relationship between particle velocity and its zeta potential. Zeta potential is defined as the electrostatic potential at the border between the diffuse layer and compact layer $<$ sup $>2</$ sup $>$ or Stern layer [24] of a colloidal system. Zeta potential is relative to surface charge of particles in suspension and is often used as an indicator of colloidal stability. The Smoluchowski approach supports that a particle's zeta potential can be determined from its velocity taking into account convective and electro osmotic forces, as well as the electrophoretic mobility of the particle. The electrophoretic mobility is a measure of the translocation time of the analyte through the pore under an applied electric field. In their seminal paper Vogel and Willmott [25] developed a method of balancing the electro osmotic and electrophoretic effects by balancing the pressure across the pore allowing zeta potential values to be extracted from the resistive pulse. In a later publication Blundell et al [26], used a similar concept that was published by Arjmandi et al [27] using pyramidal pores. Where a calibration based zeta potential method was applied, based on the measurement of signal durations of translocation events as a function of voltage. The electrophoretic mobility is calculated from the derivative of medium particle velocity and applied electric field. 
Sikora and co-workers investigated the effects of adsorbed proteins from serum on the size and surface charge of plain and aminated silica nanoparticles. The zeta potential was measured by electrophoretic light scattering (ELS) and TRPS (ref). They reported that the particle-by-particle $\zeta$-potential measurements by TRPS were consistent with those performed with ELS and allowed a description of the $\zeta$-potential distribution within the samples

\section{TRPS in analysis of nanoparticle interaction with biomolecules}

In biological fluids such as blood, the nanoparticles interact with the various constituents such as cells and plasma proteins, potentially changing the particle characteristics. Proteins in blood bind to the surface of nanoparticles to form a coating known as protein corona which can affect the interaction of nanoparticles with the biomolecules [28-29]. The protein corona formation can affect the kinetics and dynamics of the nanoparticle in biological fluids thus modifying the pharmacokinetics of nanopharmaceuticals. It is, therefore, imperative to study this phenomenon in detail.

Blundell and co-workers [30] studied the effects of plasma proteins albumin, fibrinogen and immunoglobulins on nanoparticle-protein corona formation using 100nm silica nanoparticles and TRPS. Their study showed that TRPS could monitor the kinetics of the protein corona forming on a particle and in particular what this effect then has on a nanoparticle's surface charge. The study also has shown that all three proteins had an effect on nanoparticle mobility through the pore, indicating that they are each forming protein-nanoparticle interactions. The researchers then went on to study the effects of plasma proteins on protein corona dynamics by studying changes in the zeta potential by TRPS [26]. TRPS promises to be a valuable tool to investigate this phenomenon of protein corona formation and its effect on nanoparticle kinetics in fluids, and other work by Shard et.al (ref) has compared the TRPS system with DLS for corona formation, shown in figure 2 

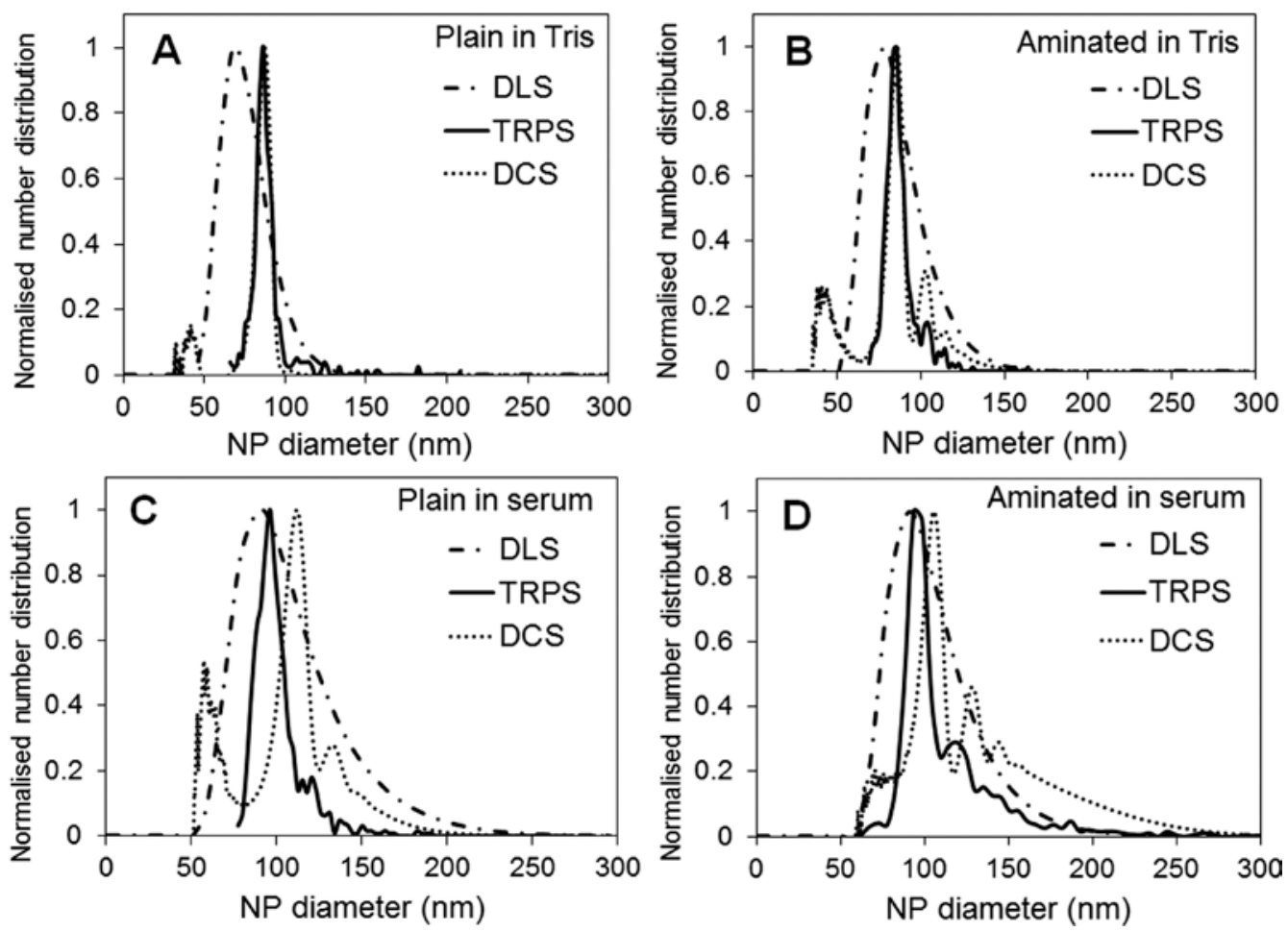

Figure 2. Normalized number-weighted particle size distributions of plain ( $A$ and $C)$ and aminated ( $B$ and $D)$ silica NPs in Tris ( $A$ and $B$ ) and serum-based ( $C$ and $D)$ medium after 24 $h$ incubation measured by DLS, DCS, and TRPS. DCS measurements are plotted assuming a densities of 2.0 and $1.125 \mathrm{~g} / \mathrm{cm} 3$ for the silica and the protein layers, respectively. Reprinted with permission from Langmuir 2016, 32, 2216-2224. Copyright (c 2016 American Chemical Society [ref]

\section{TRPS in the analysis of nanoparticle-bound DNA interactions with} biomolecules

Particle bound oligonucleotides are incorporated into many assay platforms for DNA sequencing, study of DNA-protein interactions, biosensing and drug delivery [31]. Over the last few years TRPS has been used to study the interactions between nanoparticle-bound DNA and various biomolecules by detecting changes in the particle size or surface charge due to the binding of the biomolecules to the DNA or formation of aggregates [32].

Initially TRPS was used to identify binding of a 16nm long, $48 \mathrm{kbp}$ double stranded DNA to silica nanoparticles by studying the changes in the surface charge [13]. DNA binding resulted in a reduction of surface charge without any discernible change in size. Subsequently, Booth et al [33] studied specific interaction between a 23 bp DNA fragment that was functionalised onto carboxylated dextran based magnetic particles, to its complementary target using TRPS. The hybridization of the particle bound DNA to its target sequence caused an increase in the surface charge as indicated by a rise in zeta potential.

Single Nucleotide Polymorphism (SNP) analysis forms a basis of clinical diagnosis and disease monitoring in certain diseases. An example is G487A mutation in 
glucose 6 phosphate dehydrogenase (G6PD) deficiency that causes haemolytic anaemias. Ang and Yung [34] has developed a label-free detection method to rapidly identify single base pair mismatch in G6PD deficiency. They used two sets of $25 \mathrm{~nm}$ gold particles with two different sized (18 and 100bp length) single stranded DNA fragments that are complementary to the different ends of a target attached onto them. Hybridization of the particle bound DNA with the target sequence formed different sized aggregates (dimers, trimers, tetramers and pentamers), shown in figure 3. These aggregates produced different sized pulses on TRPS. The sensitivity of this assay in detecting target DNA was between $5.0 \mathrm{pM}$ to $2.5 \mathrm{nM}$ and a result could be produced in 30 minutes.

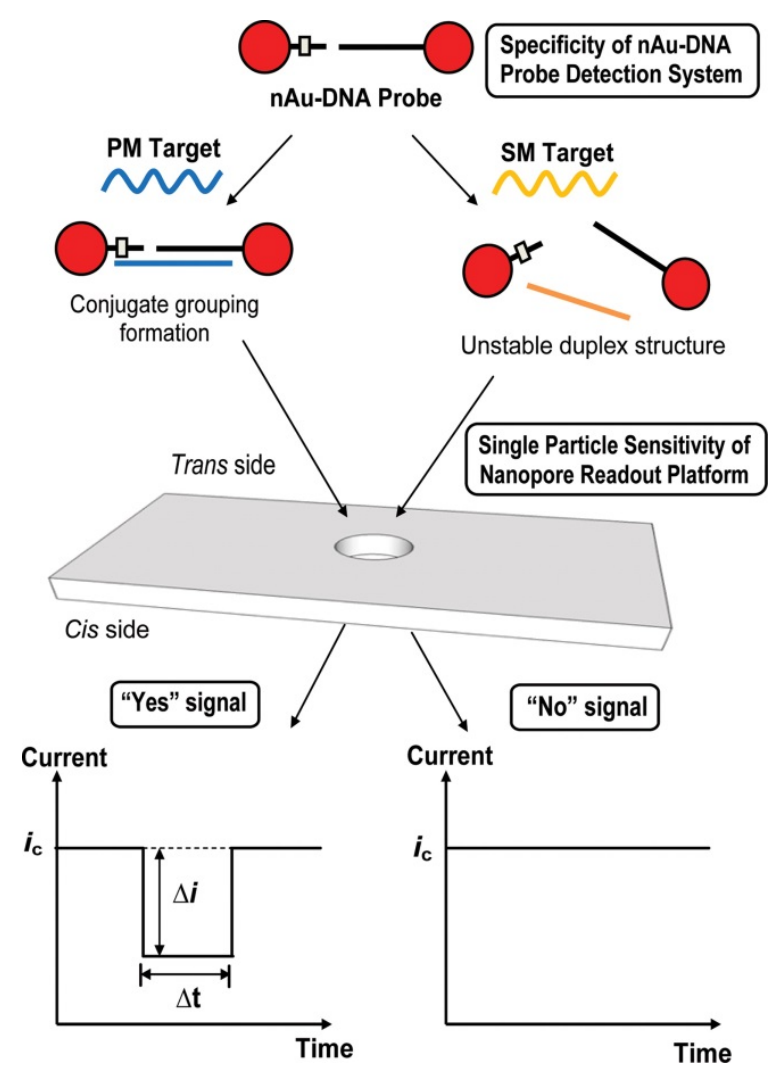

Figure 3 Schematic illustration of nanopore-based single-nucleotide detection using a $n A u-D N A$ probe. The ssDNA sequences on $n A u-100 \mathrm{~b}$ and $n A u-18 \mathrm{~b}$ probes were designed to be complementary to the mutant (mut) sequence and single mismatched to the wild-type (wt) sequence. In the presence of a perfectly matched (PM) target, a well-defined nanoparticle assembly, termed conjugate grouping, forms. Each distinct conjugate grouping is picked up as an individual signal ("Yes" signal) when it translocates the pore of the membrane from the trans to the cis side. Every successful translocation activity is termed a blockade event, which is characterized by its blockade magnitude $(\Delta i)$ and baseline translocation duration $(\Delta t)$. When a single-mismatched (SM) target is added, the intermediate duplex structure is energetically unstable and fails to form an assembly structure. The smaller-sized $n A u-D N A$ probe does not result in an appreciable dip in baseline current $\left(i_{c}\right)$ and is taken to produce a "No" signal. Reprinted with permission from ACS Nano, 2012, 6, pp 8815-8823. Copyright (2012) American Chemical Society. [ref] 
Yang et al [35] has reported a novel diagnostic assay for detection of the PantonValentine leukocidin (PVL) DNA from methicillin-resistant Staphylococcus aureus (MRSA) using TRPS and loop-mediated isothermal DNA amplification (LAMP) in combination with gold nanoparticles. The researchers first amplified the PVL DNA from MRSA by LAMP using four primers. The amplified DNA was conjugated to gold nanoparticles of two different sizes (55 and $30 \mathrm{~nm}$ respectively) to generate dual gold nanoparticle assemblies. The detection and quantification of the agglomerated gold nanoparticles were performed by TRPS. The researchers concluded that this technique is capable of detecting 500 copies of genomic DNA from the bacteria MRSA MW2 and the detection can be completed within two hours with a signal-toreadout setup providing a valuable tool for MRSA detection in clinical laboratory setting.

Kuhnemund and Nilsson [36] employed rolling circle amplification technique (RCA) to amplify the target DNA and TRPS to carry out label free detection of DNA products on magnetic particles. The target DNA molecules were detected by padlock probes which were circularised by ligase reaction. The DNA-padlock probe complex was captured on magnetic particles by sequence specific oligonucleotides and amplified by RCA. TRPS was used to detect individual particles with amplified DNA product. The lower detection limit of this technique was $1 \mathrm{fM}$ with an assay time of under one hour.

More recently, Blundell et al [37] studied the changes in zeta potential of DNA modified particles using TRPS. The high sensitivity of TRPS enabled them to resolve the signals for single stranded and double stranded DNA fragments and detect small changes in base length for oligonucleotides between 15 and 40 bases long and discriminate between partial and fully complementary target sequences. The authors note that this technique has a potential application in sensors for monitoring nanoparticles in medical and environmental samples.

TRPS in the analysis of nanoparticle bound aptamer interactions with biomolecules

Aptamers are short single-stranded pieces of DNA or RNA capable of binding to analytes with specificity and high affinity. Aptamers are generated through the process of SELEX (Systematic Evolution of Ligands by Exponential Enrichment) [3839] or by CLADE (Closed Loop Aptameric Directed Evolution) [40]. More recent technologies to incorporate aptamers as the target capture probe are Resistive Pulse Sensing [41] and its variant technology Tunable Resistive Pulse Sensing [42]. Rotem and colleagues [41] attached an aptamer for thrombin to ó-haemolysin nanopore and observed the interaction between the aptamer and its target molecule thrombin by RPS. Using this approach, they were able to detect the target molecule at nanomolar concentration. Platt and colleagues [42] were the first to utilise TRPS technology to study interactions between nanoparticle-bound aptamers and target molecules. They employed a different strategy using an aptamer for platelet derived growth factor (PDGF) tagged on to cylindrical nanorods containing gold and nickel segments. Each rod was a 'bar code'. They used TRPS to study the interaction between the aptamer and the target molecule and observed that the pulse magnitude and FWHM were specific to a particular shape of the resulting aggregate. They were able to detect the target molecule at femtomolar concentrations. 
Following on from this work, Billinge and Platt have developed a multiplexed, labelfree platform using aptamers and TRPS (AptaTRPS) for the detection of biomarkers [43]. The researchers tagged aptamers to vascular endothelial growth factor (VEGF) and platelet derived growth factor (PDGF) on to $120 \mathrm{~nm}$ and $300 \mathrm{~nm}$ streptavidin modified superparamagnetic beads respectively, shown in figure 4 . The binding of the target molecules to the aptamers was analysed by TRPS. Interestingly, they were able to observe binding of two proteins to their specific aptamers simultaneously.

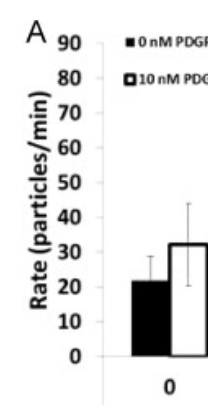

B

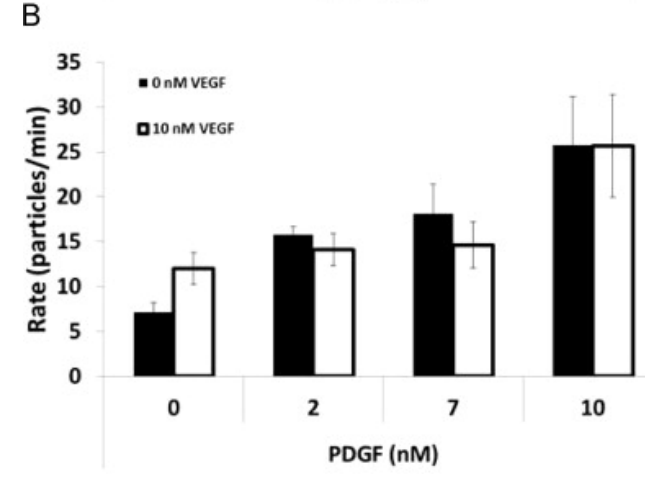

C

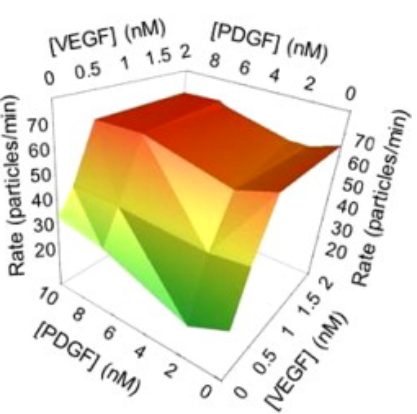

D

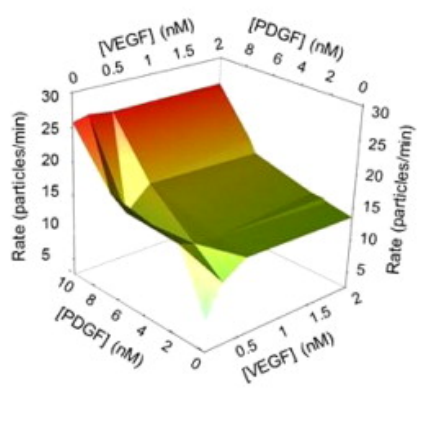

Figure 4: A bar graph comparing the average particle rates of $120 \mathrm{~nm}$ beads functionalised with V7t1 as VEGF 165 concentration increases with or without the addition of 10 nM PDGF$B B$ in the presence of $300 \mathrm{~nm}$ beads functionalised with anti-PDGF aptamer. B bar graph comparing the average particle rates of $300 \mathrm{~nm}$ beads functionalised with anti-PDGF aptamer as PDGF-BB concentration increases with or without the addition of $10 \mathrm{nM}$ VEGF 165 in the presence of $120 \mathrm{~nm}$ beads functionalised with V7t1. C 3D surface plot displaying the effect of PDGF and VEGF on particle rate of $120 \mathrm{~nm}$ beads. $D$ 3D surface plot displaying the effect of PDGF and VEGF on particle rate of $300 \mathrm{~nm}$ beads. Reprinted with permission from Biosensors and Bioelectronics, Volume 68, 2015, 741-748. [ref] 
Alsager and co-workers [44] have designed a simple sensor to detect $17 \beta$-estradiol utilising an aptamer attached to the surface of $217 \mathrm{~nm}$ sized carboxylated polystyrene nanoparticles. The interactions between the aptamer conjugated nanoparticle and the target molecule were studied by TRPS and DLS. Resistive pulse sizes increased with the attachment of the aptamer. Binding of the target to the aptamer resulted in decrease in the pulse size due to conformational change. The detection limit of this sensor system was reported to be in the lower nanomolar range.

\section{TRPS in extracellular vesicles analysis}

Extracellular vesicles (EVs) are lipid bilayer membrane vesicles secreted by most living cells. The generation and release of EVs from living cells is a physiological process that occurs during cell growth and activation without affecting cell viability [45-46]. Extracellular vesicles (ECVs) are generated either at the plasma membrane (microvesicles) or within endosomal structures (exosomes). ECVs are very heterogeneous varying greatly in size and content. They vary in size from $20 \mathrm{~nm}$ in diameter to $1000 \mathrm{~nm}$. The small ECVs $(20-100 \mathrm{~nm})$ comprise the more homogenous population of exosomes and the larger vesicles (100-1000 nm), referred to as microvesicles, originate from plasma membrane. Physiologically EVs play a role in inter-cellular communications, immune responses, coagulation, inflammation, transport, cellular homeostasis and survival. The concentration and composition of the EVs can be altered in pathological states. The EVs can carry microRNA and have been shown to promote tumour invasiveness and metastasis and can confer resistance to drugs and promote endothelial cell migration, invasion and neovascularization acting as carriers of angiogenic stimuli.

Over the last few decades, there has been an intense interest in studying EVs in order to understand their role in physiology and various pathological states including cancer, inflammatory states and neurodegenerative diseases and to explore their potential as early diagnostic and prognostic markers. Furthermore, EVs have also been explored as potential drug delivery vehicles [47]. As a result, there have been a surge in the number of diagnostic modalities to study EVs. Over the last few years, a number of studies have employed TRPS as a diagnostic tool to characterise EVs [48-52 ].

de Vrij et al [48] investigated the ability of TRPS to quantify non-purified EVs in various body fluids including urine, blood plasma and pleural fluid and culture supernatants. They found that, in all the samples studied, more than $95 \%$ of particles were in the range of $150-400 \mathrm{~nm}$. Their study confirmed that TRPS is capable of measuring MVs in biological samples without the requirement of any prior MV isolation or labelling. The reproducibility of TRPS in determining the size and concentration of EVs was studied by Coumans et al [49]. They used beads and standard urine sample for their experiment with the instrument set at fixed stretch and voltage or fixed blockade height. Based on their findings, they concluded that pore to pore variability is the cause of the variation in minimum detected size when setting a fixed stretch and voltage. The reproducibility of the minimum detectable diameter was improved by setting a fixed blockade height. 
The size distribution of MVs in the urine and blood was also studied by Cheng et al [53-54]. They undertook a study to profile miRNA in various blood components and identify differences in profiles within peripheral blood compared to cell-free plasma or serum and EVs. The characterisation of EVs was performed by TRPS. Their analysis showed that the EVs isolated from urine provided a consistent source of miRNA for further analysis.

Although a number of exosome isolation protocols are currently available, there is a significant variation in their performance. In a bid to optimise the isolation protocol for cell culture supernatant and human plasma, Lobb et al [55] has undertaken a comparative analysis of four exosome isolation techniques using TRPS and protein analysis. The results obtained from this study have enabled them to identify the current shortcomings in EV isolation methods and to propose a standardised method for EV isolation.

TRPS has also been employed to evaluate the effectiveness of therapeutic procedures in lowering certain biological substances. Effectiveness of apheresis in removing EVs was studied by combining TRPS and flow cytometry by Connolly and colleagues [56]. Their results showed that while lipoprotein-apheresis reduces circulating microparticles in patients with familial hypercholesterolaemia (FC), the procedure non-selectively lowered annexin V positive CD41 positive platelet derived particles. In another study in which nanofiltration was employed to remove microparticles to reduce thrombogenecity of human plasma, nanoparticle removal was evaluated by TRPS, DLS, NTA and flow cytometry [57].

Maas and co-workers [58] adopted a strategy of using samples spiked with polystyrene beads of known size and concentration and TRPS to measure them in order to overcome the technical hurdles encountered when measuring EVs in biological fluids. Lane et al [50] studied the effects of various exosome isolation protocols on vesicle recovery and size distribution using TRPS and 100nm liposomes as a model system, shown in figure 5. They demonstrated that liposome size distribution and $\zeta$-potential are comparable to those of extracted exosomes, making them an ideal model for comparison studies. 
A

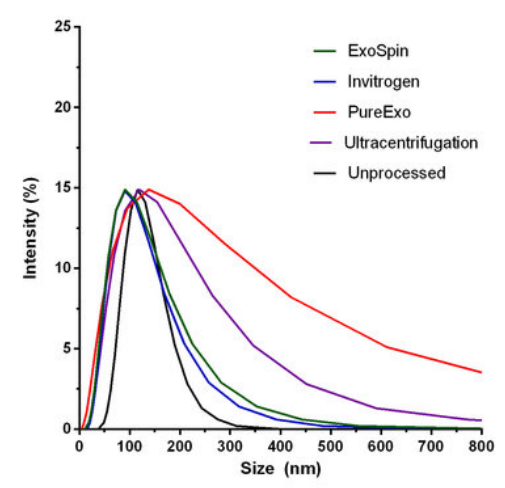

C

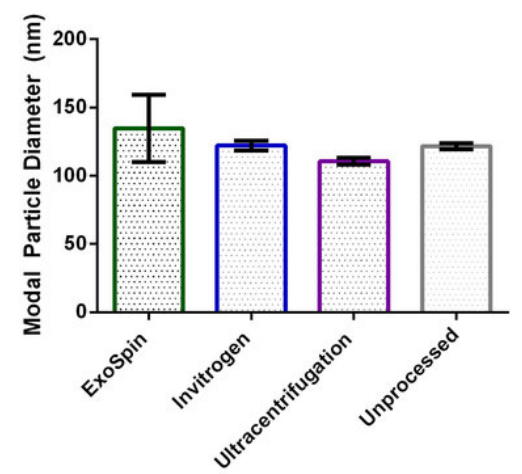

B
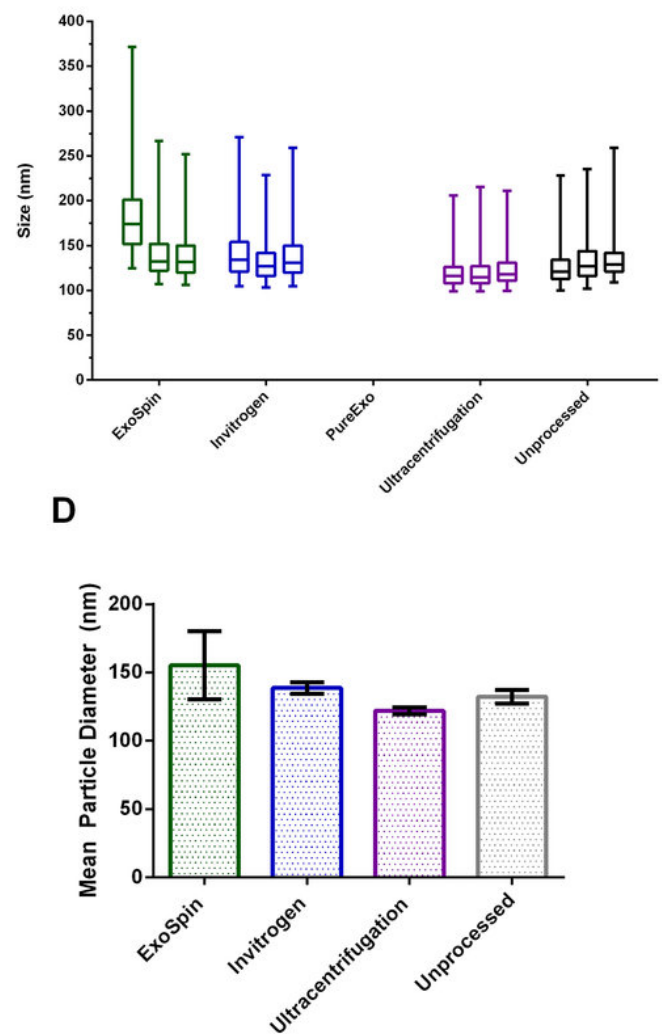

Figure 5, (A) DLS percent intensity by size $(\mathrm{nm})$ for ExoSpin, Invitrogen, PureExo, Ultracentrifugation and Unprocessed control. (B) TRPS measurements with an NP150 pore of particle size distributions for ExoSpin $(n=1777)$, Invitrogen $(n=3302)$, PureExo $(n=0)$, Ultracentrifugation $(n=923)$ and unprocessed $(n=1312)$ samples respectively. Boxes indicate 25 th, 50 th and 75 th percentile values, whiskers encapsulate 1st and 99th percentile values. (C) Modal \pm SD particle diameter for ExoSpin ( $\mathrm{n}$ $=3)$, Invitrogen $(n=3)$, ultracentrifugation $(n=3)$ treated samples and the unprocessed negative control as measured by TRPS with an NP150 pore. There was no significant difference in size between any of the processed samples and the unprocessed control ( $p>0.05)$. (D) Mean \pm SD particle diameter for ExoSpin $(n=3)$, Invitrogen $(n=3)$, ultracentrifugation $(n=3)$ treated samples and the unprocessed negative control as measured by TRPS with an NP150 pore. There was no significant difference between any of the processed samples and the unprocessed control. Reprinted with permission from Scientific Reports (2015), 5, 7639. Copyright Nature Publishing Group [ref]. 
EVs derived from malignant cells including leukaemias and solid cancers are currently studied extensively. Analysis of tumour derived EVs may offer an early diagnostic and disease monitoring tool. Increasingly TRPS is being used to characterise tumour derived EVs. Boing et al [59] studied the role of the enzyme caspase-3 in inducing microvesicle release from breast cancer cells. In their transfection study using a caspase-3 deficient breast cancer cell line, the researchers demonstrated that caspase 3 transfected cells induced production of caspase-3 containing 400-600nm sized EVs. They characterised EVs using TRPS and flow cytometry. Their study showed that, compared to flow cytometry, TRPS gave more relevant information about the size and size distribution of EVs.

Mesenchymal cell derived EVs are being studied as potential drug delivery vesicles and gene vectors. Katsuda and co-workers [60] explored the potential of EVs as drug carrier for the treatment of Alzheimer's disease which is characterised by accumulation of beta-amyloid peptide in the brain. Beta amyloid is degraded by an enzyme neprilisin. The researchers isolated neprilysin containing EVs from human adipose tissue-derived mesenchymal stem cells and characterised by TRPS and NTA. When the neprilisin expressing EVs were transferred into N2a cells, there was a reduction in the secreted and intracellular beta amyloid peptide. Shimbo et al [61] used a different strategy to obtain mesenchymal derived EVs containing synthetic miRNA (mRNA-143) for cancer therapy. They introduced the miRNA into cells and then collected the released EVs containing the synthetic RNA. They characterised the EVs by TRPS and transferred them to osteosarcoma cells. The transfer of miRNA inhibited migration of osteosarcoma cells.

It must be recognised that despite the availability of various technologies for the characterisation of EVs, there is considerable variation in the sensitivity and reproducibility between these diagnostic modalities as highlighted by the study of van der Pol [51]. The researchers measured the size and concentration of vesicles and reference beads with transmission electron microscopy (TEM), a conventional flow cytometer, a high resolution flow cytometer, nanoparticle tracking analysis (NTA), and resistive pulse sensing (RPS). Their study showed that each technique gave a different size distribution and a different concentration for the same vesicle sample. The researchers concluded that the differences between the detected vesicle concentrations are primarily caused by differences between the minimum detectable vesicle sizes. The importance of correct settings to obtain accurate and reproducible results were highlighted in the study undertaken by Mass et al [62]. They undertook a study to compare nanoparticle tracking analysis (NTA), high resolution flow cytometry and TRPS in quantifying EVs and 212nm sized synthetic liposomes. Their study showed that there were differences in absolute quantification of EVs and liposomes between the three technologies and highlighted the importance correct and standardised instrument settings to obtain accurate results. More recently, Akers et al [63] carried out a comparative analysis of technologies for quantifying extracellular vesicles (EVs) in cerebrospinal fluid of patients with glioblastoma. They compared the results obtained with TRPS, NTA, TEM and vesicle flow cytometry (VFC). Their results support the notion that the particle size does affect the quantitative analysis of EVs. For EVs $<150 \mathrm{~nm}$ in diameter, NTA detected more EVs than TRPS. For EVs $>150 \mathrm{~nm}$ diameter, NTA consistently detected lower number of EVs relative to TRPS. While TEM gave useful information about the morphology, it gave significantly lower counts compared to NTA and 
TRPS. The authors have suggested that a multiplatform quantitation will be required for clinical EV investigations.

In order to address the issue of sensitivity and stability, Anderson et al [64] has carried out a comprehensive analysis of the parameters involved in TRPS exosome measurements and demonstrated the ability to improve system sensitivity and stability by the optimisation of parameters. They also provided the first analysis of the system noise, sensitivity cut-off limits, and accuracy with respect to exosome measurements and defined the system sensitivity.

With the rapid expansion in extracellular vesicle research, it is anticipated that the utility of TRPS in characterising EVs will rise sharply over next few years.

\section{$\underline{\text { TRPS in Drug Delivery and Drug Development Applications }}$}

As alluded in the Introduction, a variety of nanoparticles including liposomes, lipid complexes, polymers, nanocrystals, inorganic nanoparticles, dentrimers and several others are being explored as potential drug delivery vehicles. Some of them are already in clinical use (eg. liposomal amphotericin, nanocrystalised tacrolimus). It is known that the physical characteristics such as size, surface charge, concentration and composition can affect the pharmakokinetics, pharmacodynamics and toxicological properties of the nanoformulations [65].

Liposomes are the most commonly used nanoparticles for drug delivery. TRPS was first applied to characterise liposomes by Garza-Licudine and colleagues [66] to quantitate liposomes and polystyrene particles ranging from 200-400nm. They demonstrated that there was a linear relationship between the capture rate (translocation events per second) and applied pressure and membrane stretching distance.

Yang et al [67] carried out a study to explore the application of scanning ion occlussion sensing (SIOS) as a novel technology for characterization of nanoparticles. The size distribution of the liposomes was measured by both SIOS and dynamic light scattering (DLS). The ability of SIOS and DLS to resolve bimodal samples was evaluated by measuring a mixture of 217 and $355 \mathrm{~nm}$ standard nanoparticles. The study showed that SIOS had higher sensitivity and better resolution than DLS in sizing bimodal particles. In addition, SIOS was able to detect opsonisation of liposomes by demonstrating an increase in the particle size and translocation rate.

$\mathrm{pH}$ responsive polymeric expansile nanoparticles are novel drug delivery vehicles (eNP) that respond to acidic environments by swelling with water and expanding. Colby et al [23] used several techniques including electron microscopy, fluorescence microscopy and TRPS to measure particle size and concentration. The study showed that eNP occurs in a continuous manner. TRPS was able to monitor the size changes that took place over time. Resistive pulse sensing has also been employed to study hydrogels, another class of drug delivery nanoformulation [68]. 
Superparamagnetic iron oxide nanoparticles (SPIONs) are currently being explored for targeted photothermal therapy in cancer. Upadhyay et al [69] investigated the potential of nanoformulation with SPION and a drug within a lipid matrix as a potentially targetable and thermosensitive inhalable drug delivery system. The therapeutic nanoparticle was characterised by TRPS. The formulations were found to be suitable for controlled delivery. The authors concluded that the system demonstrates promise as an effective drug vehicle in targeted and controlled inhalation therapy. The ability of TRPS to characterise SPIONs was also studied by Willmott and colleagues $[18,70]$. SPIONS are widely used in molecular identification, purification, separation and aggregation. These are crucial steps in drug development. On the other hand, SPIONs are increasingly used for combined in-vivo imaging and cancer therapy (so-called theranostics). These findings may suggest that the TRPS technology may have an important role in drug development and theranostics.

A number of techniques are used to characterise nanopharmaceuticals, but there is a lack of consistency and reproducibility among these technologies. In a bid to overcome these drawbacks and to satisfy the requirements of various regulatory agencies, Kozak and co-workers [72] carried out a comprehensive and high resolution analysis of liposomes. The simultaneous size and zeta potential analysis capabilities of TRPS enabled determination of the homogeneity and difference between unpegylated and pegylated liposomes.

\section{The role of TRPS in Microbiology}

The traditional methods employed to count microbes such as bacteria and viruses include microscopy (light or electron), plating and infectivity assay. These are laborious and time consuming. Hence, a rapid, simple and inexpensive method would be a great benefit to busy microbiology laboratories. In this respect, TRPS may offer a solution by providing an alternative diagnostic platform to count and size these microbes.

As discussed in the earlier section, de Blois and colleagues [7] pioneered the use of resistive pulse sensing for quantitation and sizing of viruses as early as in 1977 . But the potential of TRPS for this purpose was first explored by Willmott and colleagues [73] and Vogel and colleagues [15]. In the former study, TRPS was shown to be capable of detecting the unique shape (head and tail) of the lambda phage virus. Vogel et al used TRPS, TEM and DLS for quantitative sizing of synthetic (polystyrene) and biological (adenovirus) nanoparticles. It was shown that the calculated size of the adenovirus particle was in agreement with the results of TEM and DLS. Subsequently, Roberts and co-workers [19] used tunable pore technology to measure concentrations of Baculovirus. The determined concentrations agreed with those obtained from microscopic counting and flow cytometry.

Arjmandi et al [74] used the technique to measure the mass of nanoparticles and viruses. They were able to measure the size and density of human immunodeficiency virus and Epstein-Barr virus. Using vesicular stomatitis virus (VSV) and 100nm polymer spheres as size controls, Akpinar \& Yin [75] has shown that TRPS was able to quantify concentrations down to 107 particles per $\mathrm{ml}$ which 
was 50 fold lower than the detection limit of TEM. TRPS produced more reproducible counts than TEM. Furthermore, the total-to-infectious particle ratio of VSV populations as measured by TRPS and plaque assay has shown that each VSV particle was infectious. In addition, TRPS provided useful information about the particle size distributions based on hundreds of particles.

TRPS has also been explored as a tool to measure concentration and size of bacteria. Roberts et al [19] were the first to apply TRPS to measure bacteria. They determined concentrations of marine photosynthetic cyanobacterium Prochlorococcus in the same experiment with Baculovirus. The results were comparable to those of microscopic analysis and flow cytometry.

Yu and colleagues [76] have used TRPS for accurate and sensitive monitoring of bacterial growth. Using Bacillus subtilis and Escherichia coli, the researchers compared the sensitivity of detection of TRPS with the standard methods of bacterial growth detection, namely colony plating and optical density. Their results showed that TRPS is sensitive and accurate relative to the standard methods with a lower detection limit of $5 \times 10^{5}$ cells $/ \mathrm{ml}$. In addition, there was general agreement between TRPS and microscopy in cell volume measurements. The ability of TRPS to generate information about the concentration and the volume of the bacterial cell simultaneously may provide valuable information about the physical aspects of cell dynamics in real time.

TRPS has also been applied for characterisation of bacteria in dairy products such as probiotics [77] and in environmental science [78-79]. In the study of Chung and co-workers [78], they used TRPS to measure the size distribution and surface charge of Francisella tularensis in their investigation of biofilm formation whereas Pang and colleagues [79] carried out comparative analysis of the size distribution and surface charge of biotin and glycoprotein coated microspheres to that of Cryptosporidium parvum oocytes in a bid to develop surrogate markers to quantify removal of the oocytes in porous media.

Heider and Metzner [80], in their review of four modalities (TRPS, NTA, flow cytometry and field flow fractionation-multiple angle laser light scattering (FFFMALLS) for single virus particle analysis, have highlighted the advantages and disadvantages of these techniques. The authors noted that the TRPS determined virus particle concentration was several fold higher than the infectivity of the virus.

\section{TRPS in Nanotoxicology}

While nanopharmaceuticals offer great promise for targeted delivery of drugs and in theranostics, there are genuine concerns about their safety for in-vivo applications. Hence, it is paramount that detailed characterisation of nanoparticles are carried out to minimise any potential toxicity when they are introduced in to the human body. Pal and colleagues [81] investigated the utility of TRPS for characterisation of four different types of nanoparticles (oxidised single walled carbon nanohorns, carbon black, cerium oxide and nickel nanoparticle) in cell culture media containing serum. The performance of TRPS was compared to that of DLS. Their results shown in figure 6 below, showed that although the size distribution obtained by the two 
techniques were comparable, TRPS offered higher resolution and sensitivity compared to DLS and unique insights into size distribution and concentration of the nanomaterials, as well as particle behavior and morphology in complex media

\section{Dynamic Light Scattering}

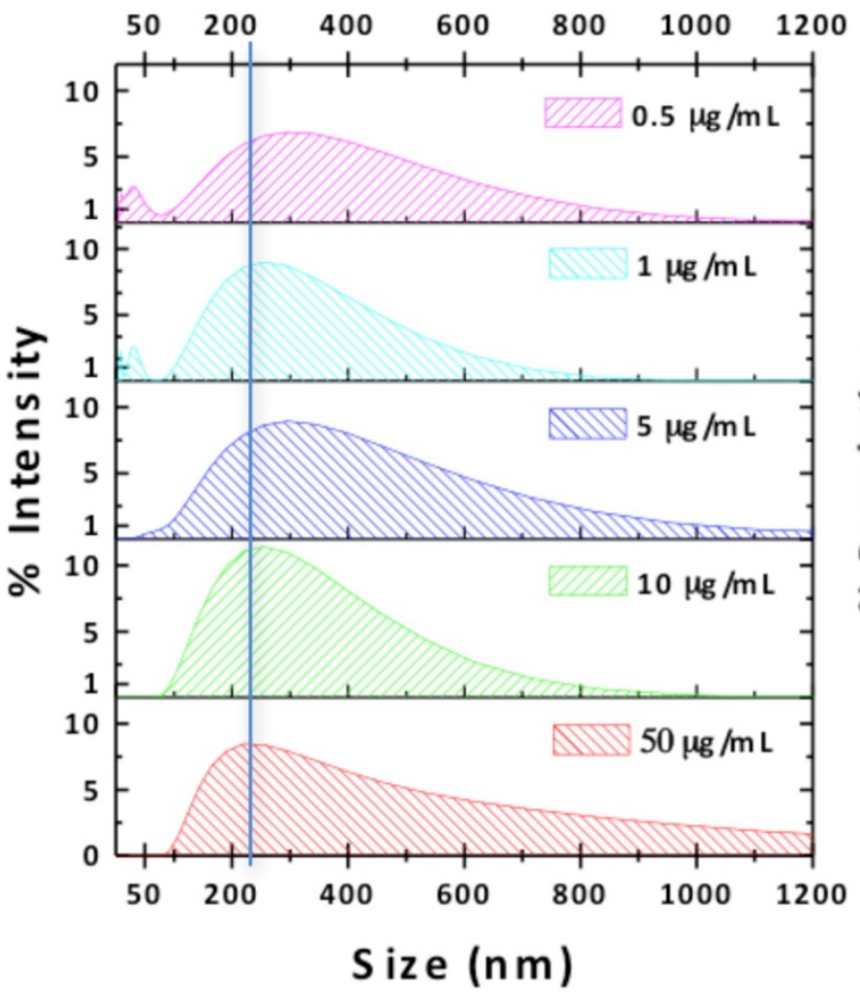

Tunable Restive Pulse Sensing

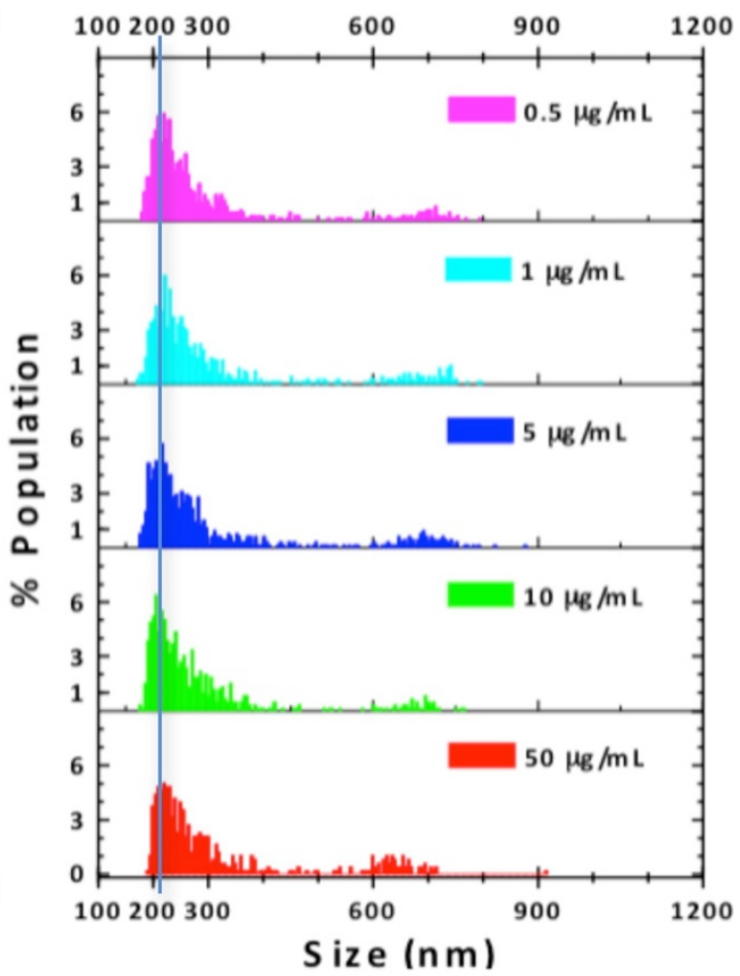

Figure 6. Comparative evaluation of TRPS and DLS in characterizing sensitivity and stability of size distribution measurements of a series of sequential dilutions of SWCNH-ox in the range of 0.5-50 $\mu \mathrm{g} / \mathrm{mL}$, prepared from a stock solution of $500 \mu \mathrm{g} / \mathrm{mL}$ in RPMI+10\% FBS. The graphs represent averages of triplicate measurements. Note changes in the DLS size distributions below $5 \mu \mathrm{g} / \mathrm{mL}$, especially left-side broadening of the peak and appearance of a smaller peak $<50 \mathrm{~nm}$, related to proteins in serum. At higher concentrations $(50 \mu \mathrm{g} / \mathrm{mL})$ the peak broadened to the right, In contrast to DLS, the TRPS size distribution remained fairly constant over the whole concentration range. Reprinted with permission from ACS Nano 2014, 8, 9003-9015. Copyright (c) 2014 American Chemical Society [ref]

One of the methods by which nanopharmaceutical toxicity on blood cells such as erythrocytes, leucocytes and platelets could be studied is by analysing the membrane changes of that cell population. Since red cells are the most abundant cells in the body, toxicological studies of these cells may provide valuable information about the toxicity of the nanoparticle under investigation. Cheung et al [82] investigated the early apoptotic changes of erythrocytes, the so called 'eryptosis', in response to a cytotoxic agent spolyphyllin D. The researchers studied the cell shrinkage and translocation of phosphatidylserine (PS) to the outer lipid bilayer of the plasma membrane, a hallmark of apoptosis, using microbeads functionalised with annexin-V for PS binding. The results of their study showed that this strategy of combining annexin- $V$ beads and TRPS is a sensitive, reliable and simple technique to determine eryptosis of human erythrocytes for various biomedical applications. 


\section{Conclusion and Future Perspective}

TRPS promises to be a valuable tool for nanoparticle characterisation. The ability of this technique to carry out particle by particle measurement of size and surface charge in addition to determining the concentration of polydisperse solutions and colloids makes it particularly useful for analysing nanopharmaceuticals. This technique permits studying nanoparticle behaviour in various biological fluids such as blood and plasma, in particular, the important phenomenon of protein corona formation. In addition, this technique can also be used to study the pharmacological and toxicological effects of effects of nanopharmaceuticals on blood cells. This application may become useful in evaluating nanopharmaceuticals prior to various regulatory approval processes.

In the rapidly expanding field of extracellular vesicle research, TRPS is proving a useful technique to size exosomes. An accurate determination of the size distribution of EVs may facilitate understanding of the biological and pathological role of these vesicles in physiology and in various disease states. It may also aid development of exosome based drug/gene delivery systems.

TRPS also has a potential for the development of a rapid and low cost platform for quantification and characterisation of microbes including bacteria and viruses. The ability of TRPS to perform tagless detection of subtle changes in the size, shape and surface charge may enable development of biosensors using organic/inorganic nanoparticles, DNA, aptamers and other nanomaterials to detect biological molecules for early diagnosis of diseases and to monitor therapeutic responses.

This technique also has a potential in toxicological evaluation of nanoparticles by providing valuable information about the interactions between the nanoparticles and blood components such as proteins and cells. This technique may, therefore, aid various regulatory authorities in evaluating nanopharmaceuticals for clinical applications.

While the above mentioned potentials are recognised, it must also be emphasised that the technology of TRPS requires optimisation and standardisation to establish sensitivity, specificity and reproducibility. The manufacturers of the TRPS based devices and their users must conduct detailed, systematic and comparative evaluation of various technologies that are currently used for nanoparticle characterisation. 


\section{Executive Summary}

Nanoparticle characterisation is an important pre-requisite for developing various nanodiagnostic platforms and, more importantly, nanopharmaceuticals

A diverse array of measurement modalities ranging from microscopy to diffraction techniques and spectroscopies are currently in use. No single technique can reliably be used to carry out the necessary measurements of different nanomaterials that are being studied for nanomedicine applications.

Tunable Resistive Pulse Sensing (TRPS) is a recent addition to the list of nanomaterial characterisation techniques. It is a variant of the established technique of Resistive Pulse Sensing (RPS) invented by Coulter in 1953. A size tunable elastomeric pore membrane is used to change the pore size and to improve detection sensitivity.

One of the important features of TRPS is its ability to measure simultaneously the size and surface charge of individual nanoparticles. It is also capable of analysing polydispersed nanoparticle samples and colloids.

This technology may provide a rapid and low cost platform to count and characterise bacteria and viruses.

The ability of TRPS in detecting minute changes in the size, surface charge and shape permits development of sensitive biosensors by using nanomaterials such as organic/inorganic nanoparticles, DNA and aptamers.

TRPS is proving a valuable tool in the rapidly expanding field of extracellular vesicle research by providing accurate information about the size distribution of exosomes that are generated in physiological and various pathological conditions including cancer and inflammatory diseases.

The ability of TRPS to study the interactions between nanomaterials and blood components such as plasma proteins and cells may permit development of assay platforms to evaluate nanopharmaceuticals for clinical use.

To realise the full potential of this technology, it needs optimisation and standardisation and also comparative analysis with other available technologies to precisely define its role in nanomedicine and nanotechnology. 


\section{$\underline{\text { References }}$}

1] Freitas RA. What is nanomedicine? Nanomedicine, 1(1):2-9 (2005).

2] Jain KK. Nanotechnologies. In 'The Handbook of Nanomedicine' (Second Edition). Edited by Jain KK. pp 7-56. Human Press, New York.

3] Logothetidis S. Nanomedicine: The Medicine of Tomorrow. In 'Nanomedicine and Nanobiotechnology'. Edited by Logothetidis S. pp 1-24. Springer, New York (2011).

4] Nel AE, Madler L, Velegol D et al. Understanding biophysicochemical interactions at the nano-bio interface. Nat Mater, 8(7), 543-57 (2009).

5] Kulkarni SK. Analysis Techniques. In 'Nanotechnology: Principles and Practices' (Third Edition). pp 135-198. Springer, New York (2015).

6] Coulter WH. Means for counting particles in a fluid. US patent 2,656,508 (20 October 1953). ${ }^{\prime * * 1}$ A ground breaking invention of Resistive Pulse Sensing technology that paved the way for the development of numerous particle counters including blood cell analysers widely used in medicine.

7] de Blois RW \& Wesley KA. Sizes and Concentrations of Several Type C Oncornaviruses and Bacteriophage T2 by the Resistive-Pulse Technique. J. Virol, 23, 227-233 (1977).

8] Kasianowicz JJ, Brandin E, Branton D et al. Characterization of individual polynucleotide molecules using a membrane channel. Proc. Natl. Acad. Sci. USA, 93, 13770-13773 (1996). ). "**' A landmark publication that re-ignited interest in Resistive Pulse Sensing technology

9] Henriquez RR, Ito T, Sun L, Crooks RM. The resurgence of Coulter counting for analyzing nanoscale objects. Analyst, 129, 478-482 (2004).

leh OA, Sohn LL. Direct detection of antibody-antigen binding using an on-chip artificial pore. Proc Natl Acad Sci USA. 2003;100(3):820-4

Lan WJ, Holden DA, Zhang B et al. Nanoparticle transport in conical-shaped nanopores. Anal Chem. 2011;83(10):3840-7.\#

Sexton LT, Horne LP, Martin CR. Developing synthetic conical nanopores for biosensing applications. Mol Biosyst. 2007;3(10):667-85.

10] Clarke J, Wu HC, Jayasinghe $L$ et al. Continuous base identification for singlemolecule nanopore DNA sequencing. Nat Nanotechnol, 4(4), 265-70 (2009).

11] Kozak D, Anderson W, Vogel R et al. Advances in Resistive Pulse Sensors: Devices bridging the void between molecular and microscopic detection. Nano 
Today, 6:531-545 (2011). "**' Comprehensive review article on the evolution of Resistive Pulse Sensing and TRPS and its principles

12] Dekker C. Solid-state nanopores. Nat Nanotechnol, 2(4), 209-15 (2007).

13] Roberts GS, Kozak D, Anderson $W$ et al. Tunable nano/micropores for particle detection and discrimination: scanning ion occlusion spectroscopy. Small, 6(23), 2653-8 (2010).

14] Willmott GR, Vogel R, Yu SS et al. Use of tunable nanopore blockade rates to investigate colloidal dispersions. J Phys Condens Matter, 22(45), 454116 (2010).

15] Vogel R, Willmott G, Kozak D et al. Quantitative sizing of nano/microparticles with a tunable elastomeric pore sensor. Anal Chem, 83(9), 3499-506 (2011).

16] Vogel $\mathrm{R}$, Anderson $\mathrm{W}$, Eldridge $\mathrm{J}$ et al. A variable pressure method for characterizing nanoparticle surface charge using pore sensors. Anal Chem, 84(7), 3125-31 (2012).

17] Willmott GR, Moore PW. Reversible mechanical actuation of elastomeric nanopores. Nanotechnology, 19(47), 475504 (2008).

18] Willmott GR, Platt M, Lee GU. Resistive pulse sensing of magnetic beads and supraparticle structures using tunable pores. Biomicrofluidics, 6(1), 14103-15 (2012).

19] Roberts GS, Yu S, Zeng Q et al. Tunable pores for measuring concentrations of synthetic and biological nanopore dispersions. Biosens Bioelectron, 31(1), 17-25 (2012).

20] Sowerby SJ, Petersen GB, Broom MF, Jones MD, Sowerby S, Petersen G, Broom M, Jones M. Patent. WO2006063872-A1; Sowerby SJ, Broom MF, Petersen GB. Sens.Actuators, B, 2007, 123, 325. "**' Pioneering work that introduced the concept of 'tunability' to Resistive Pulse Sensing technology

21] Willmott GR, Parry BET. Resistive pulse asymmetry for nanospheres passing through tunable submicron pores. J. Appl. Phys. 109 (2011).

22] Kozak D, Anderson W, Trau M. Tuning Particle Velocity and Measurement Sensitivity by Changing Pore Sensor Dimensions. Chem. Lett, 41, 1134-1136 (2012).

23] Colby $\mathrm{AH}$, Colson $\mathrm{YL}$, Grinstaff MW. Microscopy and tunable resistive pulse sensing characterization of the swelling of $\mathrm{pH}$-responsive, polymeric expansile nanoparticles. Nanoscale, 5(8), 3496-504 (2013).

24] Kaszuba $M$, Corbett $\mathrm{J}$, Watson $\mathrm{FM}$ et al, High-concentration zeta potential measurements using light-scattering techniques. Philos. Trans. A. Math. Phys. Eng. Sci, 368, 4439-51 (2010).

25] Vogel $\mathrm{R}$, Anderson $\mathrm{W}$, Eldridge $\mathrm{J}$ et al. A variable pressure method for characterizing nanoparticle surface charge using pore sensors. Anal Chem, 84(7), 3125-31 (2012). 
26] Blundell E, Holton E, Healey et al. Characterisation of the Protein Corona using Tunable Resistive Pulse Sensing: Determining the Change and Distribution of a Particles Surface Charge. 2016 (Submitted for publication).

27] Arjmandi, N., Van Roy, W., Lagae, L. \& Borghs, G. Measuring the electric charge and zeta potential of nanometer-sized objects using pyramidal-shaped nanopores. Anal. Chem. 84, 8490-8496 (2012).

28] Tenzer S, Docter D, Kuharev J et al. Rapid formation of plasma protein corona critically affects nanoparticle pathophysiology. Nat Nanotechnol, 10, 772-81 (2013).

29] Pozzi D, Caracciolo G, Digiacomo $L$ et al. The biomolecular corona of nanoparticles in circulating biological media. Nanoscale, 7(33), 13958-66 (2015).

30] Blundell E, Holton E, Healey et al. A study of surface interactions between carboxyl polystyrene nanoparticles and various human blood components using tunable resistive pulse sensing. 3rd International Conference in Nanotechnology in Medicine, Manchester, UK (November 2015).

31] Tokel O, Inci F, Demirci U. Advances in plasmonic technologies for point of care applications. Chem Rev, 114(11), 5728-52 (2014).

32] Weatherall E, Willmott GR. Applications of tunable resistive pulse sensing. Analyst, 140, 3318-3334 (2015).

33] Booth MA, Vogel R, Curran JM et al. Detection of target-probe oligonucleotide hybridization using synthetic nanopore resistive pulse sensing. Biosens. Bioelectron, 45, 136-40 (2013).

34] Ang YS, Yung LY. Rapid and label-free single-nucleotide discrimination via an integrative nanoparticle-nanopore approach. ACS Nano. 6(10), 8815-23 (2012).

35] Yang AK, Lu H, Wu SY et al. Detection of Panton-Valentine Leukocidin DNA from methicillin-resistant Staphylococcus aureus by resistive pulse sensing and loopmediated isothermal amplification with gold nanoparticles. Anal Chim Acta, 782: 4653 (2013).

36] Kuhnemund $M$, Nilsson $M$. Digital quantification of rolling circle amplified single DNA molecules in a resistive pulse sensing nanopore. Biosens Bioelectron, 67, 1117 (2015).

37] Blundell EL, Vogel R, Platt M. Particle-by-Particle Charge Analysis of DNAModified Nanoparticles Using Tunable Resistive Pulse Sensing. Langmuir. 32(4)1082-90 (2016).

38] Tuerk C, Gold L. Systematic evolution of ligands by exponential enrichment: RNA ligands to bacteriophage T4 DNA polymerase. Science, 249(4968), 505-10 (1990).

39] Ellington $A D$, Szostak JW. Selection in vitro of single-stranded DNA molecules that fold into specific ligand-binding structures. Nature, 355(6363), 850-2 (1992). 
40] Knight CG, Platt M, Rowe W et al. Array-based evolution of DNA aptamers allows modelling of an explicit sequence-fitness landscape. Nucleic Acids Res, 37(1), e6.doi:10.1093 (2009).

41] Rotem D, Jayasinghe $L$, Salichou $M$ et al. Protein detection by nanopores equipped with aptamers. J Am Chem Soc, 134(5), 2781-7 (2012).

42] Platt $M$, Willmott GR, Lee GU. Resistive pulse sensing of analyte-induced multicomponent rod aggregation using tunable pores. Small, 8(15), 2436-44 (2012).

43] Billinge ER, Platt M. Multiplexed, label-free detection of biomarkers using aptamers and Tunable Resistive Pulse Sensing (AptaTRPS). Biosens Bioelectron. 68, 741-8 (2015).

44] Alsager OA, Kumar S, Willmott GR et al. Small molecule detection in solution via the size contraction response of aptamer functionalized nanoparticles. Biosens Bioelectron. 57, 262-8 (2014).

45] Cocucci E, Racchetti G, Meldolesi J. Shedding microvesicles: artefacts no more. Trends Cell Biol, 19, 43-51 (2009).

46] Raposo G, Stoorvogel W. Extracellular vesicles: exosomes, microvesicles, and friends. J Cell Biol, 200(4), 373-83 (2013).

47] El Andaloussi S, Mager I, Breakefield XO et al. Extracellular vesicles: biology and emerging therapeutic opportunities. Nat Rev Drug Discov, 12(5), 347-57 (2013).

48] de Vrij J, Mass SL, van Nispen M et al. Quantification of nanosized extracellular membrane vesicles with scanning ion occlusion sensing. Nanomedicine, 8(9), 144358 (2013).

49] Coumans FA, van der Pol E, Boing AN et al. Reproducible extracellular vesicle size and concentration determination with tunable resistive pulse sensing. J Extracell Vesicles, 3, 25922 (2014).

50] Lane RE, Korbie D, Anderson W et al. Analysis of exosome purification methods using a model liposome system and tunable-resistive pulse sensing. Sci Rep, 5, 7639 (2015).

51] Van der Pol E, Coumans FA, Grootemaat AE et al. Particle size distribution of exosomes and microvesicles determined by transmission electron microscopy, flow cytometry, nanoparticle tracking analysis, and resistive pulse sensing. $\mathrm{J}$ Thromb Haemost, 12(7), 1182-92 (2014).

52] Van der Pol E, Boing AN, Gool EL et al. Recent developments in the nomenclature, presence, isolation, detection and clinical impact of extracellular vesicles. J Thromb Haemost, 14(1), 48-56 (2016).

53] Cheng L, Sharples RA, Scicluna BJ et al. Exosomes provide a protective and enriched source of miRNA for biomarker profiling compared to intracellular and cellfree blood. J Extracell Vesicles. 3(10) 3402/jev.v3.23743 (2014). 
54] Cheng L, Sun $X$, Scicluna BJ et al. Characterization and deep sequencing analysis of exosomal and non-exosomal miRNA in human urine. Kidney Int, 86(2), 433-44 (2014).

55] Lobb RJ, Becker M, Wen SW et al. Optimized exosome isolation protocol for cell culture supernatant and human plasma. J Extracell Vesicles, 4, 27031 (2015).

56] Connolly KD, Willis GR, Datta DB et al. Lipoprotein-apheresis reduces circulating microparticles in individuals with familial hypercholesterolemia. J Lipid Res, 55(10), 2064-72 (2014).

57] Chou ML, Lin LT, Devos D, Bu et al. Nanofiltration to remove microparticles and decrease the thrombogenecity of plasma: in vitro feasibility assessment. Transfusion, 55 (10), 2433-44 (2015).

58] Maas SL, de Vrij J, Broekman ML. Quantification and size-profiling of extracellular vesicles using tunable resistive pulse sensing. J Vis Exp, 92, e51623 (2014).

59] Boing AN, Stap J, Hau CM et al. Active caspase-3 is removed from cells by release of caspase-3-enriched vesicles. Biochim Biophys Acta. 1833(8),1844-52 (2013).

60] Katsuda T, Tsuchiya $\mathrm{R}$, Kosaka $\mathrm{N}$ et al. Human adipose tissue-derived mesenchymal stem cells secrete functional neprilysin-bound exosomes. Sci Rep, 3, 1197 (2013).

61] Shimbo K, Miyaki S, Ishitobi H et al. Exosome-formed synthetic microRNA-143 is transferred to osteosarcoma cells and inhibits their migration. Biochem Biophys Res Commun, 445(2), 381-7 (2014).

62] Maas SL, de Vrij J, van der Vlist EJ et al. Possibilities and limitations of current technologies for quantification of biological extracellular vesicles and synthetic mimics. J Control Release, 200, 87-96 (2015).

63] Akers JC, Ramakrishnan V, Nolan JP et al. Comparative Analysis of Technologies for Quantifying Extracellular Vesicles (EVs) in Clinical Cerebrospinal Fluids (CSF). PLoS. 11(2), e0149866 (2016).

64] Anderson W, Lane R, Korbie $\mathrm{D}$ et al. Observations of Tunable Resistive Pulse Sensing for Exosome Analysis: Improving System Sensitivity and Stability. Langmuir. 31(23), 6577-87 (2015).

65] Torchilin VP. Nanocarriers. Pharm Res, 24, 2333-4 (2007).

66] Garza-Licudine E, Deo D, Yu S et al. Portable nanoparticle quantization using a resizable nanopore instrument - the IZON qNano ${ }^{\mathrm{TM}}$. Conf Proc IEEE Eng Med Biol Soc, 437(1-2), 253-63 (2010).

67] Yang L, Broom MF, Tucker IG. Characterization of a nanoparticulate drug delivery system using scanning ion occlusion sensing. Pharm Res, 29(9), 2578-86 (2012). 
68] Pevarmik $M$, Schiel $M$, Yoshimatsu $K$ et al. Particle deformation and concentration polarization in electroosmotic transport of hydrogels through pores. ACS Nano, 7(4), 3720-8 (2013).

69] Upadhyay D, Scalia S, Vogel $\mathrm{R}$ et al. Magnetised thermo responsive lipid vehicles for targeted and controlled lung drug delivery. Pharm Res, 29(9), 2456-67 (2012).

70] Willmott GR, Fisk MG, Eldridge J. Magnetic microbead transport during resistive pulse sensing. Biomicrofluidics, 7(6), 64106 (2013).

72] Kozak D, Broom M, Vogel R. High resolution particle characterization to expedite development and regulatory acceptance of nanomedicines. Curr Drug Deliv, 12(1), 115-20 (2015).

73] Willmott GR, Broom MF, Jansen ML, Young RM, Arnold WM. In 'Molecular and Nanotubes', ed O.Hayden and K Nielsch, Springer, New York, ch.7, pp 209-261 (2011).

74] Arjmandi N, Van Roy W, Lagae L. Measuring mass of nanoparticles and viruses in liquids with nanometer-scale pores. Anal Chem. 86, 4637-41 (2014).

75] Akpinar F, Yin J. Characterisation of vesicular stomatitis virus populations by tunable resistive pulse sensing. J Virol Methods. 218, 71-76 (2015).

76] Yu AC, Loo JF, Yu S et al. Monitoring bacterial growth using tunable resistive pulse sensing with a pore-based technique. Appl Microbiol Biotechnol, 98(2), 855-62 (2014).

77] Bennett G, Rajan R, Bunt CR et al. Microbiological assessment of four probiotic feed supplements used by the dairy industry in New Zealand. NZ Vet J. 61(2), 11920 (2013).

78] Chung M-C, Dean S, Marakasova ES et al. Chitinases are negative regulators of Francisella novicida biofilms. PLoS One, 9(3), e93119 (2014).

79] Pang L, Nowostawska U, Weaver L. Biotin- and glycoprotein-coated microspheres: potential surrogates for studying filtration of cryptosporidium parvum in porous media. Environ Sci Technol. 2012;46(21):11779-87.

80] Heider S, Metzner C. Quantitative real-time single particle analysis of virions. Virology, 462, 199-206 (2014).

81] Pal AK, Aalaei L, Gadde $S$ et al. High resolution characterization of engineered nanomaterial dispersions in complex media using tunable resistive pulse sensing technology. ACS Nano, 8(9), 9003-15 (2014).

82] Cheung AKL, Yang AKL, Ngai BH et al. Quantitative detection of eryptosis in human erythrocytes using tunable resistive pulse sensing and annexin-V-beads. Analyst, 140, 1337-1348 (2015). 